\title{
On Demand Network Level Service Deployment in Ad Hoc Networks
}

\author{
Daniel Prince, Andrew Scott, and W.D. Shepherd \\ DMRG, Computing Department Lancaster University, \\ Lancaster, LA1 4YR, UK \\ $\{$ d.prince, acs, doug $\} \varrho_{c}$ comp. lancs.ac.uk
}

\begin{abstract}
Many network level services such as routing, auto-addressing and, security have been proposed for ad hoc networks. Each has characteristics that may cause it to outperform similar services under different network conditions; therefore the choice of optimal services depends on the network deployment. Furthermore, since ad hoc network groups may be mobile it is probable that networks operating different or varying service implementations will interact. This paper presents work to provide dynamic, on demand network service selection and deployment, with minimal user interaction, in ad hoc networks.
\end{abstract}

\section{Introduction}

The demand for ad hoc network technology is growing as computing becomes increasingly pervasive and mobile. The IETF Mobile Ad hoc NETwork (MANET) Working Group [4] and wider research community have produced designs for various network level services such as routing protocols [9, 11], auto-addressing [7], and gateway protocols [2]. Simulations [3, 8] show that routing protocols perform optimally for particular network characteristics (node mobility/density). We expect all classes of network level service to display this property. With heterogeneous network level services, it is difficult to predict the services present at ad hoc network formation and inefficient to provide them on all devices. Additionally, some may be suboptimal for current networking conditions making it important to select the most appropriate.

As with Packet Radio Networks [10] before them, ad hoc networks require predetermination of network services in order to interact. However, technologies such as PCMCIA/compact flash or Software Defined Radio (SDR) allow wireless interfaces to be dynamically selected and deployed "on the fly" to suit conditions. We believe that similar capabilities for network level services would greatly enhance the adaptability and self organisational properties of ad hoc networks and that Programmable Networking provides a suitable basis for an appropriate solution.

In this paper, we discuss our motivating scenario and a real world example. We then present a new node mobility model, used in the design of a solution, which captures the scenarios behaviour. We go on to present our system design and finally conclude with a discussion on further and related work. 


\section{Scenario}

The 'squad and base' model is the name given to our generic scenario. It comprises two main elements, large base and small squad ad hoc networks. Nodes in both networks are equipped with a common type of wireless interface. Base ad hoc networks are formed by large groups of users, typically around 100+, who ordinarily have very low mobility levels. Therefore, the resultant ad hoc network of mobile nodes has a fairly stable network topology. Squad ad hoc networks are much smaller, 5 to 10 nodes, and display increased dynamicity over base networks. The typical mobile-temporal relationships of these two types of networks are shown in Figure 1.

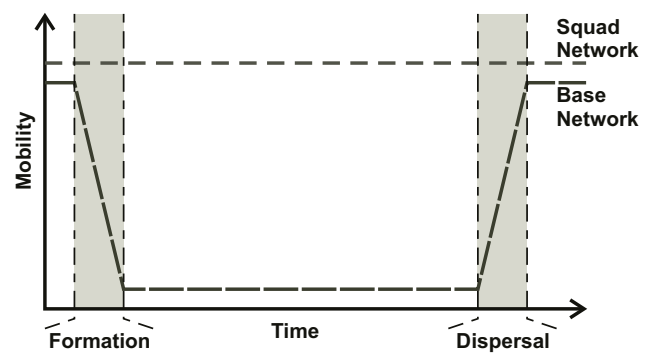

Fig. 1. Mobile Temporal Relationships of a Squad and Base Network.

\subsection{Real World Example}

Consider a scenario in which base networks are lecture groups using real-time multimedia applications and squad networks are student groups around a campus sharing files and information. Here, service selection is not only based on network characteristics but also application constraints. To reduce latency the real-time application requires timely route creation, suggesting a proactive routing protocol such as OLSR [1]. In contrast, file sharing has minimal latency constraints suggesting an on-demand protocol such as DSR [9] or AODV [11].

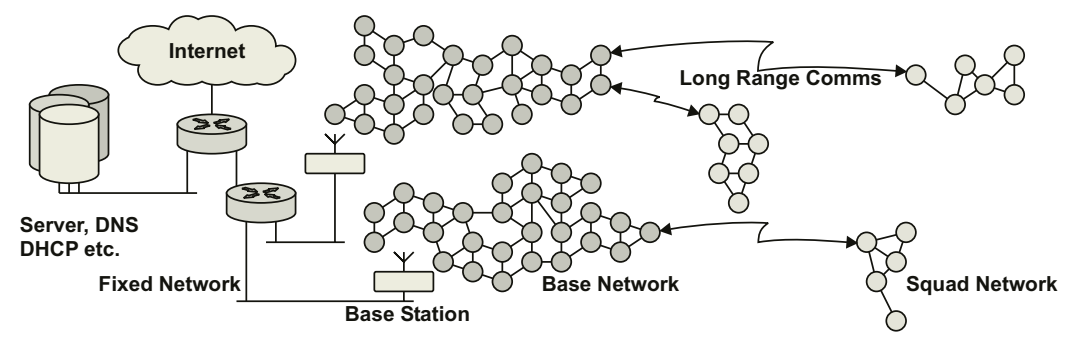

Fig. 2. Complete Scenario Diagram

The convergence of a student and lecture group raises an initial problem as the potential lack of common network level services will make network interaction and inter-network node migration difficult. Network separation, through lecture group dispersal, presents additional problems as previously common lecture group network 
level services may have characteristics that cause sub-optimal performance or in extreme cases network failure, in the newly formed student groups. So far only autonomous networks have been discussed. However, connectivity to high speed fixed network infrastructures need to be considered. Here, mobile nodes that provide interconnectivity between LAN and ad hoc networks may need additional services such as gateway protocols and auto-addressing. Fig. 2 shows the overall scenario.

\section{Dynamic Group Mobility}

In developing the scenario depicted in Figure 2, the authors concluded that the scenario presented complex network interactions which existing node mobility models [6,9] do not fully capture. As a result, suitable system design parameters and constraints could not be taken from simulations based upon these models. This led to the development of the Dynamic Group Mobility (DGM) model.

DGM is based on the Group Mobility (GM) model presented in [6]. Both GM and DGM groups have a central point which moves through a way point set and an area in which nodes move. However, inside this area DGM nodes move with a Random Waypoint behaviour [9] where as GM nodes move around randomly distributed fixed points. Additionally, DGM nodes are free to change group membership.

On initialisation each node is given random associativity and fickle values between 0 and 100. Group changes occur when a node detects a neighbour which is in a different group. The nodes then examine their associativity values to determine whether they should stay together. If they should, then the fickle factor is used to decide which node should change groups. Figure 3 gives the algorithm. To compensate for changes in group size, the group radius changes proportionally to the number of nodes in the group.

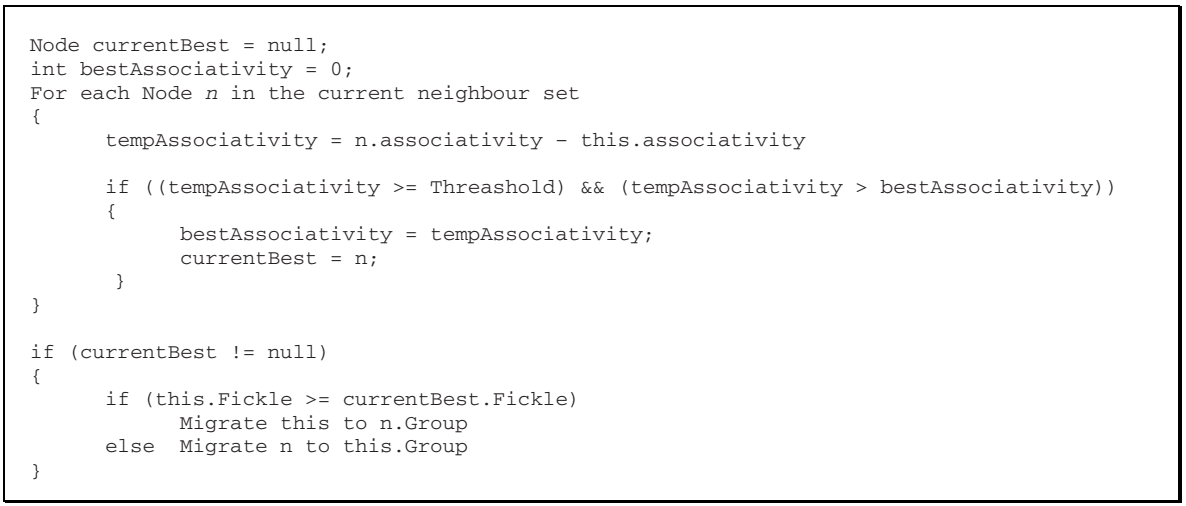

Fig. 3. Migration algorithm pseudo code.

A simulator has been implemented in $\mathrm{C \#}$ to evaluate node behaviour with the various models and is available for download. Statistical analysis of interaction type and duration are being used as design parameters for algorithms and protocols used in the ANOM service described in the following section. 


\section{System Design and Implementation}

Many ad hoc network level protocols and services must manipulate packets at various points in the processing path. For example, DSR inserts and processes source route data while MobileIP based gateway protocols [2] manipulate header options. In many Active Network platforms, installed services access packets using dynamic packet filtering. The system under development is based on the Lancaster Active Router Architecture (LARA++) [12]. In LARA++ received data traverses a dynamic Classification Graph. At each classification node services install filters in order to receive packets. LARA++ also allows services to be dynamically instantiated.

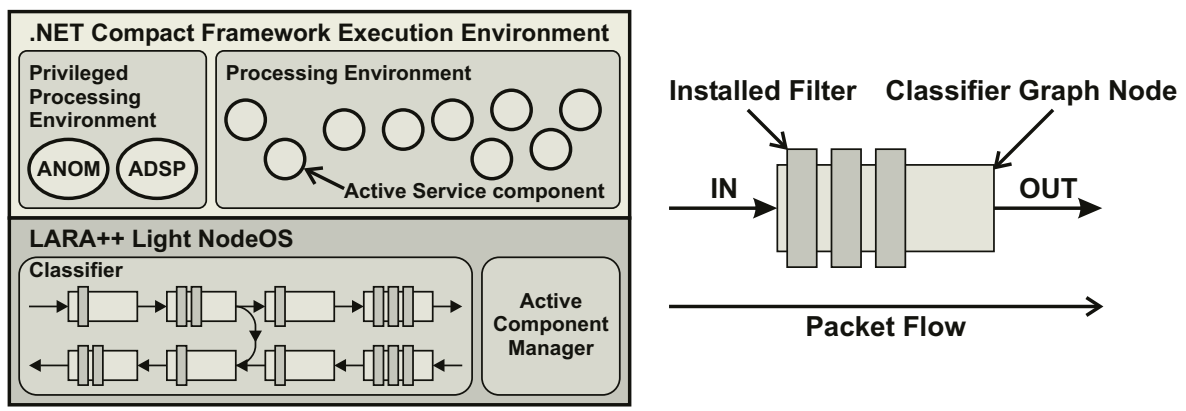

Fig. 4. System design with a classification node diagram

The system design, shown in Figure 5, consists of three main elements:

1. LARA++ Light - A lightweight version of LARA++, based on WinCE and the .NET compact framework, capable of running on limited hardware such as PDAs.

2. Active Service Discovery Protocol (ASDP) [13] - Allows clients to query and publish services on active nodes.

3. Ad hoc Network Observer and Manager (ANOM) - ANOM is responsible for monitoring network characteristics, distributing/evaluating service characteristics and then deciding with rest of the network the best service to deploy.

Network level services are then implemented as active components.

To demonstrate node bootstrapping and continued operation, consider a mobile device with LARA++ light and ASDP attempting to access an ad hoc network. Network nodes running the ANOM component can detect whether an ANOM component is instantiated on the new node. If not, a network neighbour can use ADSP to request the node install the ANOM component. Subsequently the new node can retrieve other services from its neighbours to bootstrap itself into the network. Additionally, if a node arrives with new services, the ANOM components would distribute information about those services, evaluate their characteristics against various constraints and potentially elect to run a new service deemed more optimal for the given environment. 


\section{Further Work}

The following three main areas have yet to be fully addressed in this work.

\subsection{Security}

Existing security mechanisms for active networks, such as code signing, static code checks and sandboxing, software fault isolation can protect an active node from malicious or buggy services attempting to disrupt its operation. However, these mechanisms cannot prevent misbehaviour within allowed system constraints such as providing incorrect results, i.e. routes. Such misbehaviour may cause extreme network degradation, making recovery exceedingly difficult. To this end a node must be capable of assessing service performance to determine intentional misbehaviour or whether current network characteristics are outside its nominal operational limits.

\subsection{Service Characteristics}

To be able to characterise services, benchmark parameters for each class of service must be identified. Simulation work $[3,8]$ and the MANET WG have already defined benchmark parameters such as Packet Delivery Ratio for routing protocols. Similar parameters for other services are yet to be defined. An extensible metadata language must be defined so that service benchmark parameters and other variables may be formed into service descriptions similar to the one in Figure 6. Note that .NET framework assemblies include an augmentable XML metadata code description.

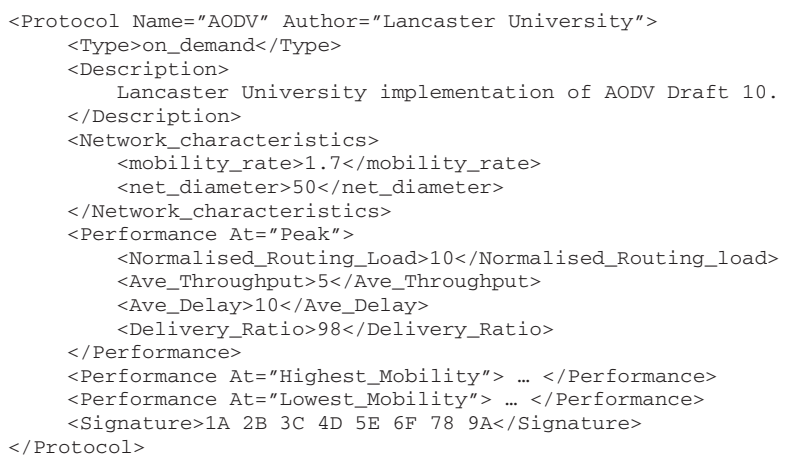

Fig. 6. An example XML service description

\subsection{Distributed Service Evaluation}

As described previously the ANOM component is responsible for evaluating service characteristics against the current network state and selecting the most suitable service. This must be a distributed operation as every node must migrate to the new service in order to maintain network connectivity. This means that the ANOM components must be capable of conversing, evaluating and voting on different service metadata descriptions in a distributed, stable and scalable manner. 


\section{Related Work and Conclusions}

Using programmable networking for routing in ad hoc networks has been, and still is being researched. SoftNET [5] was an early active node developed for routing in PRNs. More recently underlay networks [15] and modular versions of existing routing protocols [14] have been implemented using programmable networking.

This work examines the wider issue of interoperability in heterogeneous ad hoc environments and aims to provide a platform to enable nodes to flexibly and optimally deploy network level services on demand. Additionally, the requirements of network level services form a strong argument for an active network based solution.

This paper has presented the squad and base scenario which we believe provides a strong argument for adaptive, on demand network level service selection and deployment in ad hoc networks. The Dynamic Group Mobility model is introduced as a mechanism to inform the development of a suitable solution. Finally, a work in progress system has been presented based on an existing active network platform.

\section{References}

1. Adjih C. et al.: Optimized Link State Routing Protocol (OLSR), IETF Draft 2003, draftietf-manet-olsr-09.txt, (April 2003), (Work in Progress).

2. Belding-Royer, E. et al.: Global Connectivity for IPv4 Mobile Ad hoc Networks. IETF Draft, draft-royer-manet-globalv4-00.txt, (Nov, 2001), (Work in Progress).

3. Broch, J. et al.: A Performance Comparison of Multihop Wireless Ad Hoc Network Routing Protocols. Proc. IEEE/ACM MOBICOM '98, (Oct, 1998).

4. Corson, S. and, Macker, J.: Mobile Ad Hoc Networking (MANET): Routing Protocol Performance Issues and Evaluation Considerations, RFC 2501 (1999).

5. Forchheimer R. and Zander J.: Softnet - Packet Radio in Sweden. In Proceedings of AMRAD Conference, (1981).

6. Hong X. et al.: A Group Mobility Model for Ad Hoc Wireless Networks, In Proceedings of ACM/IEEE MSWiM'99, Seattle, WA, (Aug, 1999).

7. Jeong, J et al.: Ad Hoc IP Address Autoconfiguration, IETF Draft, draft-jeong-adhoc-ipaddr-autoconf-00.txt, (May 2003), (Work in Progress).

8. Johansson, P. et al.: Scenario-Based Performance Analysis of Routing Protocols for Mobile Ad-Hoc Networks. Proceedings of MOBICOM'99, (Aug, 1999).

9. Johnson, D. and Maltz, D.: Dynamic source routing in ad hoc wireless networks, in Mobile Computing (ed. T. Imielinski and H. Korth), Kluwer Academic (1996).

10. Jubin J. and Tornow J.: The DARPA Packet Radio Network Protocols, The proceedings of the IEEE, Vol. 75, No. 1, (Jan, 1987).

11. Perkins, C. et al.: Ad Hoc on Demand Distance Vector (AODV) Routing. draft-ietfmanet-aodv-10.txt, IETF Draft, (2002), (Work In Progress).

12. Schmid, S. et al.: Component-Based Active Network Architecture. Proceedings of 6th IEEE Symposium on Computers and Communications, (2001).

13. Sifalakis M. et al.: A Generic Active Service Deployment Protocol. To appear in the proceedings of ANTA2003, (May 2003).

14. Tornquist Modular and Adaptive routing in ad hoc networks, Masters thesis Department of Computer Science, University of Colorado, (2001).

15. Tschudin and R. Gold, "LUNAR: Lightweight Underlay Network Ad-Hoc Routing," tech. rep., University of Basel, Switzerland, (Jan, 2002). 